\title{
Correction to: Ideals, Varieties, and Algorithms
}

\section{Correction to: \\ D.A. Cox et al., Ideals, Varieties, and Algorithms, Undergraduate Texts in Mathematics, https://doi.org/10.1007/978-3-319-16721-3}

After initial publication of the book, various errors were identified that needed correction. The following corrections have been updated within the current version, along with all known typographical errors.

\section{Chapter 1}

Page 23, part (c) of Exercise 5: "Adapt the argument given at the end of the section" should be "Adapt the argument used for the circle $x^{2}+y^{2}=1$ "

Page 24, line 1: "adapt the argument given at the end of the section" should be "adapt the argument used for the circle $x^{2}+y^{2}=1$ "

Page 39, line 6 of the paragraph beginning "To see why this algorithm works": "By (5)", should be "By (1)," \footnotetext{
https://doi.org/10.1007/978-3-319-16721-3_1 https://doi.org/10.1007/978-3-319-16721-3_2 https://doi.org/10.1007/978-3-319-16721-3_3 https://doi.org/10.1007/978-3-319-16721-3_4 https://doi.org/10.1007/978-3-319-16721-3_5 https://doi.org/10.1007/978-3-319-16721-3_6 https://doi.org/10.1007/978-3-319-16721-3_7 https://doi.org/10.1007/978-3-319-16721-3_8 https://doi.org/10.1007/978-3-319-16721-3_9 https://doi.org/10.1007/978-3-319-16721-3_10 https://doi.org/10.1007/978-3-319-16721-3
}

The updated online versions of these chapters can be found at 
Page 47, part (a) of Exercise 14: "where $h(a) \neq 0$ " should be "where $r \geq 1$ and $h(a) \neq 0$ "

\section{Chapter 2}

Page 63, bottom display, second line underneath $\overline{:}:$ " $x y^{2}-x$ " should be " $x^{2} y-x$ "

Page 63, bottom display, fourth line underneath $\overline{\quad}:$ " $x^{2} y-y$ ” should be " $x y^{2}-y$ "

Page 66, lines -5 and -6 : "[using condition (ii) of the definition of a monomial order]" should be "[using Lemma 8 of $\$ 2]$ "

Page 81, line 1 of Exercise 2: "LT $(I)$ " should be " $\langle\mathrm{LT}(I)\rangle$ "

Page 82, last line of Exercise 13: "Exercise 14 of Chapter 1, §4” should be "Proposition 8 of Chapter $1, \S 4$ "

Page 89, part (d) of Exercise 5: " $z^{2}-3$ " should be " $z^{2}-3 z$ "

Page 93, line -3: “ $\langle\operatorname{LT}(G \backslash\{p\})\rangle$ ” should be “ $\langle\operatorname{LT}(G \backslash\{g\})\rangle$ ”

Page 96, line 2 of part (a) of Exercise 12: "is not divisible by" should be "has leading term not divisible by"

Page 97, line 3 of Exercise 14: “ $\frac{x_{j}-a_{j}}{a_{i}-a_{j}}$ ” should be “ $\frac{x-a_{j}}{a_{i}-a_{j}}$ ”

Page 105, lines 13-16: Replace $x y+1$ with $x y-1$ in three places and $-x-y$ with $-x+y$ in two places. Thus the lines should be as follows:

$\S 3$. If we divide $f=x y^{2}-x$ by $G=\left(x y-1, y^{2}-1\right)$, the division algortihm gives

$$
x y^{2}-x=y \cdot(x y-1)+0 \cdot\left(y^{2}-1\right)+(-x+y)
$$

so that $\bar{f}^{G}=-x+y \neq 0$. Yet we can also write

$$
x y^{2}-x=0 \cdot(x y-1)+x \cdot\left(y^{2}-1\right),
$$

Page 107, line -4 : " $f_{3}=x z+y-z+1$ " should be " $f_{3}=x z-x+y+1$ "

Page 108, Exercise 1: " $f_{3}=x z+y-z+1$ " should be " $f_{3}=x z-x+y+1$ "

Page 110, Lemma 4: The statement of the lemma should be changed to the following:

Lemma 4. Every element of $S(F)$ can be written as a sum of homogeneous elements of $S(F)$. Furthermore, this decomposition is unique.

Page 115, line 12 "by Lemma 2" should be "by Lemma 2 of $\S 9$ ” 


\section{Chapter 3}

Page 136, line 20: " $(t, u, x, y, z) \in \mathbf{V}(I) \subseteq \mathbb{R}^{5 "}$ should be " $(t, u, x, y, z) \in \mathbf{V}(I) \subseteq$ $\mathbb{C}^{5}$ "

Page 140, Exercise 3: " $t^{2}$ is always positive" should be " $t^{2}$ is always $\geq 0$ "

Page 154, part (c) of Exercise 15: The $x$-coordinate of the second displayed point should be

$$
\pm \frac{1}{2} \sqrt{15+6 \sqrt[3]{2}-12 \sqrt[3]{4}}
$$

Page 160, part (b) of Exercise 4: " $g_{\mathrm{o}}=g_{3}$ " should be " $g_{\mathrm{o}}=g_{2}$ "

Page 167, line -17: Replace "It follows that" with "(Proposition 5 applies to $f, g$ since their coefficients lie in the field $\left.k\left(x_{2}, \ldots, x_{n}\right)\right)$. It follows that"

Page 169, line -7: " $u_{1}\left(x_{1}\right)$ " should be " $u\left(x_{1}\right)$ "

Page 170, line 1: " $\left\langle f_{i}, f_{*}\right\rangle$ " should be " $\left\langle f_{i}, f^{*}\right\rangle$ "

Page 170, part (c) of Exercise 3: "part (a) is still true but part (b) can fail" should be "parts (a) and (b) are still true"

Page 171, line 2 of Exercise 9: " $l \geq m$ " should be " $l \geq m>0$ "

\section{Chapter 4}

Page 180, line 13: "must have $f_{i}\left(a_{1}, \ldots, a_{n}\right)=0$ " should be "must have $f_{i}\left(a_{1}, \ldots, a_{n}\right) \neq 0$ "

Page 181, lines 1 and 2 of Exercise 10: " $\mathbb{R}[x, y]$ " should be " $\mathbb{R}[x, y, z]$ " in two places.

Page 181, line 4 of Exercise 10: "same for $\mathbb{R}[x]$ " should be "same for $\mathbb{R}[x]$ and $\mathbb{R}[x, y]^{\prime}$

Page 187, line -3: " $a_{1} \frac{\partial f_{i}}{\partial x_{j}} h_{i}$ " should be " $a_{i} \frac{\partial f_{i}}{\partial x_{j}} h_{i}$ "

Page 189, Exercise 15: Replace the hint with "Hint: Show that $x y, x z, y z$ generate the ideal of leading terms of $\sqrt{I}$ and use the definition of Gröbner basis given in Chapter 2, §5."

Page 196, line 4: "principal ideals is principal)" should be "two principal ideals is principal)"

Page 206. Exercise 5: Replace the hint with "Hint: Examine the generators of $J^{s M}$."

Page 212, Exercise 10: "Theorem 11 implies" should be "Theorem 11 and Proposition 6 of $\$ 7$ imply"

Page 221, line 1: "by Exercise 3 of Chapter 2, §9" should be "by Exercise 15" 
Page 221, Corollary 3: "With the same notation" should be "With $k$ algebraically closed and the same notation"

Page 222, line 4: "for all $i$ " should be "for all such $i$ "

Page 222, line 5 of Proposition 5: "a variety contained in $V$ " should be "a variety contained in $\mathbf{V}\left(I_{l}\right)$ ",

Page 223, line -17: " $W \subsetneq \mathbf{V}(I)$ " should be " $W \subsetneq \mathbf{V}\left(I_{l}\right)$ "

Page 223, line -9: "fails for $I, \mathbf{V}(I) \backslash$ " should be "fails for $I, \mathbf{V}\left(I_{l}\right) \backslash$ "

Page 223, line -8: "by Proposition 4" should be "by Proposition 4 (we can assume $G$ is reduced)"

Page 224, second display: The display should be as follows:

$$
\mathbf{V}\left(I_{1}\right) \backslash \mathbf{V}\left(c_{1}\right)=\mathbb{C} \backslash \mathbf{V}(y)=\mathbb{C} \backslash\{0\} \subseteq \pi_{1}(\mathbf{V}(I)) \subseteq \mathbf{V}\left(I_{1}\right)=\mathbb{C}
$$

Page 228: Add the following new exercise:

15. In the setting of Theorem 2, prove that $\mathbf{x}^{\gamma}>\operatorname{LT}(f)$ implies $\mathbf{x}^{\gamma}>\operatorname{LT}(\bar{f})$ for $f \in k[\mathbf{x}, \mathbf{y}]$.

Page 231, line 9: “EXERCISES FOR §9” should be “EXERCISES FOR §8”

Page 231, part (b) of Exercise 11: "Exercise 4" should be "Exercise 6"

Page 231, Exercise 12: "Use Proposition 9 of §4” should be "Use Exercise 4 of $₫ 4$ ”

\section{Chapter 5}

Page 247, line 1: " $R=k[x, t]$ " should be " $R=k[t]$ "

Page 247, line 2 of part (b) of Exercise 10: " $a, b \in k[x]$ " should be " $a, b \in k$ "

Page 256, Exercise 6: "Let $V=\mathbf{V}\left(x_{3}-x_{1}^{2}, x_{4}-x_{1} x_{2}, x_{2} x_{4}-x_{1} x_{5}, x_{4}^{2}-x_{3} x_{5}\right) \subseteq \mathbb{C}^{5}$ " should be "Let $V=\mathbf{V}(I) \subseteq \mathbb{C}^{5}$ for $I=\left\langle x_{3}-x_{1}^{2}, x_{4}-x_{1} x_{2}, x_{2} x_{4}-x_{1} x_{5}, x_{4}^{2}-x_{3} x_{5}\right\rangle \subseteq$ $\mathbb{C}\left[x_{1}, x_{2}, x_{3}, x_{4}, x_{5}\right]$."

Page 256, line -1: At the end, add "Assume that the field $k$ is infinite."

Page 257, part (e) of Exercise 11: "we developed in Chapter 1" should be "we developed in Chapter 1, §2"

Page 260, lines 2-4: These three lines

(iii) is proved in the same way as Theorem 11 of Chapter $4, \S 5$.

When $k$ is algebraically closed, the Weak Nullstellensatz also holds in $k[V]$. You will prove this in Exercise 16. 
should be replaced with the following:

(iii) is proved by first showing that the Weak Nullstellensatz also holds in $k[V]$. You will prove this in Exercise 16. From here, one proceeds in the same way as Theorem 11 of Chapter $4, \S 5$.

Page 271, line following second display: " $\mathbf{V}_{W}\left(a^{2}-b^{2}+4\right)$ " should be " $\mathbf{V}_{W}\left(y^{2}-\right.$ $\left.z^{2}+4\right) "$

Page 272, second paragraph of the proof of Proposition 6: In two places, "V $\left(f_{i} g_{i}^{\prime}-\right.$ $\left.f_{i}^{\prime} g_{i}\right)$ " should be "V $V\left(f_{i} g_{i}^{\prime}-f_{i}^{\prime} g_{i}\right)$ "

Page 279, display (1): Replace the display with

$$
s^{\ell}+c_{1} s^{\ell-1}+\cdots+c_{\ell}=0, \quad c_{1}, \ldots, c_{\ell} \in R .
$$

Page 279, line - 10: " $a_{i \ell} s_{i \ell}$ " should be " $a_{i \ell} s_{\ell}$ "

Page 279, line -7: "the coefficient of $x$ is" should be "the coefficient of $x^{\ell}$ is"

Page 279, the last display should be:

$$
\operatorname{det}\left(A-x I_{\ell}\right)=(-1)^{\ell}\left(x^{\ell}+c_{1} x^{\ell-1}+\cdots+c_{\ell}\right) .
$$

Page 279, line -4: " $a_{i} \in R$ " should be " $c_{i} \in R$ "

Page 280, line 4: " $C$ has entries in $R$ " should be " $C$ has entries in $S$ "

Page 280, line -13: “(ii) $\Rightarrow$ (iii)" should be “(i) $\Rightarrow$ (iii)"

Page 280, line -9: "divide $f$ by $G$ " should be "divide $f$ by a Gröbner basis $G$ "

Page 282, line -6: "finite over $k[y]$ " should be "finite over $k\left[y_{1}, \ldots, y_{m}\right]$ "

Page 288, line 2 of Exercise 6: "means geometrically" should be "means geometrically when $k$ is algebraically closed"

Page 288, line 1 of Exercise 13: "in (4) is" should be "in (5) is"

Page 288, line 2 of part (a) of Exercise 13: "the substitution (5)" should be "the substitution (4)"

Page 289, last line of part (a) of Exercise 17: “ $\phi(\mathbf{V}(J))$ " should be “ $\pi(\mathbf{V}(J))$ "

\section{Chapter 6}

Page 294, line below second display: " $C=U \times V$ " should be " $\mathcal{C}=U \times V$ "

Page 300, first display: " $f\left(\theta_{1}+\theta_{2}+\theta_{3}\right)$ " should be " $f\left(\theta_{1}, \theta_{2}, \theta_{3}\right)$ "

Page 302, line 2: “ $\mathcal{J}=\mathbf{V}\left(x_{1}^{2}+y_{1}^{2}-1, x_{2}^{2}+y_{2}^{2}-1, x_{3}^{2}+y_{3}^{2}-1\right)$ " should be $" \mathcal{J}=\mathbf{V}\left(c_{1}^{2}+s_{1}^{2}-1, c_{2}^{2}+s_{2}^{2}-1, c_{3}^{2}+s_{3}^{2}-1\right) "$ 
Page 302, two lines below (7): " $V=\mathbf{V}\left(x_{1}^{2}+y_{1}^{2}-1, x_{2}^{2}+y_{2}^{2}-1, x_{3}^{2}+y_{3}^{2}-1\right)$ " should be " $V=\mathbf{V}\left(c_{1}^{2}+s_{1}^{2}-1, c_{2}^{2}+s_{2}^{2}-1, c_{3}^{2}+s_{3}^{2}-1\right)$ "

Page 304, part (a) of Exercise 9: "result of part (c)" should be "result of part (e)"

Page 305 , line 12: "in equation (7) of $\S 2$ " should be "in equation (6) of $\S 2$ "

Page 305, line 1 of (2): “ $\frac{2 b l_{2} l_{3}}{2 l_{2}\left(a^{2}+b^{2}\right)} s_{2}$ ” should be “ $\frac{b l_{2} l_{3}}{l_{2}\left(a^{2}+b^{2}\right)} s_{2}$ ”

Page 305, line 2 of (2): “ $\frac{2 a l_{2} l_{3}}{2 l_{2}\left(a^{2}+b^{2}\right)} s_{2}+$ " should be “ $\frac{a l_{2} l_{3}}{l_{2}\left(a^{2}+b^{2}\right)} s_{2}-$ "

Page 306, line 1 of (3): “ $\frac{2 b}{2\left(a^{2}+b^{2}\right)} s_{2}$ ” should be “ $\frac{b}{a^{2}+b^{2}} s_{2}$ ”

Page 306, line 2 of (3): “ $\frac{2 a}{2\left(a^{2}+b^{2}\right)} s_{2}+$ " should be “ $\frac{a}{a^{2}+b^{2}} s_{2}-$ "

Page 307, line -3: "when $a^{2}+b^{2}<4$," should be "when $0<a^{2}+b^{2}<4$,"

Page 308, line 6: "if $l_{4}$ lies in" should be "if $l_{2}=l_{3}=1$ and $l_{4}$ lies in"

Page 308, line 8 of the subsection Specialization of Gröbner Bases: “ $k\left[x_{1}, \ldots, x_{m}\right.$, $\left.t_{1}, \ldots, t_{m}\right]$ " should be " $k\left[x_{1}, \ldots, x_{n}, t_{1}, \ldots, t_{m}\right]$ "

Page 309, display in the middle of the page: Replace the display with

$$
1, l_{2}, l_{3}, l_{2}, l_{3}, 1, l_{2} l_{3}, l_{2} l_{3}, l_{2} l_{3}, a, b, a^{2}+b^{2}, l_{2} l_{3} \text {. }
$$

Page 309, two lines below display: “ $a, b, l_{2}, l_{3}, a^{2}+b^{2}$ and $a^{2}+b^{2}-l_{2}^{2}-l_{3}^{2}$ are nonzero" should be " $a, b, l_{2}, l_{3}$ and $a^{2}+b^{2}$ are nonzero"

Page 312, line 3: "We have" should be "When $l_{2}=l_{3}=1$, we have"

Page 316, line 5: " $B_{i j} \in k(\mathbf{t})[\mathbf{x}]$ ” should be " $B_{j i} \in k(\mathbf{t})[\mathbf{x}]$ ”

Page 316, part (c) of Exercise 7: Replace the hint with "Hint: The monomial orders for $k(\mathbf{t})[\mathbf{x}]$ and $k[\mathbf{x}]$ are the same-the parameters $t_{j}$ are "constants" as far as the ordering is concerned. Theorem 6 of Chapter 2, $\$ 9$ will be useful."

Page 316, lines 7 and 8 of Exercise 8: Delete these lines and replace them with the following:

nonzero polynomials $F_{i}$ and $G_{j}$ in $k[\mathbf{t}]$, we get

$$
\tilde{f}_{i}=F_{i} f_{i}, \tilde{g}_{j}=G_{j} g_{j} \in k[\mathbf{x}, \mathbf{t}] .
$$

Page 316, line 9 of Exercise 8: " $\tilde{I} \subseteq k(\mathbf{t})[\mathbf{x}]$ " should be " $\tilde{I} \subseteq k[\mathbf{x}, \mathbf{t}]$ " 
Page 316, part (a) of Exercise 8: Replace part (a) with the following:

a. Fix $j$ and suppose $g_{j}=\sum_{i=1}^{s} B_{j i} f_{i}$ in $k(\mathbf{t})[\mathbf{x}]$ and let $d_{j} \in k[\mathbf{t}]$ be a polynomial that clears the denominators of $B_{j 1}, \ldots, B_{j s}$. Also let $F=\operatorname{lcm}\left(F_{1}, \ldots, F_{s}\right)$. Then prove that

$$
d_{j} \in\left(\tilde{I}: F \tilde{g}_{j}\right) \cap k[\mathbf{t}],
$$

where $\tilde{I}: F \tilde{g}_{j}$ the ideal quotient as defined in $\S 4$ of Chapter 4 .

Page 316, part (b) of Exercise 8: “( $\left.\tilde{I}: \tilde{g}_{j}\right) \cap k[\mathbf{t}]$ ” should be “( $\left(\tilde{I}: F \tilde{g}_{j}\right) \cap k[\mathbf{t}]$ "

Page 317, part (b) of Exercise 11: " $c_{i} \in \mathbb{R}\left[a, b, l_{2}, l_{3}\right]$ " should be " $h_{i} \in \mathbb{R}\left[a, b, l_{2}, l_{3}\right]$ "

Page 326, line 4 of the first display should be:

$$
f_{4}=x_{3} u_{3}+x_{4} u_{1}-x_{4} u_{2}-u_{1} u_{3},
$$

Page 331, part (b) of Exercise 2: Replace with "b. With this choice, explain why we can specify the coordinates of $B$ as $B=\left(u_{3}, 0\right)$, i.e., the $x$-coordinate of $B$ is arbitrary, but the $y$-coordinate is zero."

Page 332, Exercise 10: "made in Example 1" should be "made in the continuation of Example 1"

Page 332, line -1: "reducible components" should be "irreducible components"

Page 333, line 2 of part (e) of Exercise 14: "follows from part (a)" should be "follows from part (b)"

Page 333, line 2 of part (e) of Exercise 14: “ $(c \cdot g)$ 's" should be “ $(c \cdot g)$ " "

Page 333, line 3 of part (c) of Exercise 15: "show that $\bar{c}$ has" should be "show that $c \bar{c}$ has"

Page 336, lines 6-7: Interchange the order of these two lines. Thus the line " $q:=$ $\ldots$ ". should be above the lines " $r:=\ldots$ "

\section{Chapter 7}

Page 348, line -6: "LT $\left(\sigma_{1} \sigma\right)$ " should be “LT $\left(\sigma_{1} \sigma_{2}\right)$ "

Page 354 , first display of Exercise 11: " $h_{j-i}\left(x_{k}, \ldots, x_{n}\right)$ " should be " $h_{j-i}\left(x_{j}, \ldots, x_{n}\right)$ "

Page 355, line 1 of the display in Exercise 15: “+ $+(-1)^{j-1} \sigma_{k-1} x_{i}+(-1)^{j} \sigma_{k}=$, should be " $+(-1)^{j-1} \sigma_{j-1} x_{i}+(-1)^{j} \sigma_{j}=$ "

Page 355, display of Exercise 18: " $=s_{j}=\sigma_{1} s_{j-1}+$ " should be " $=s_{j}-\sigma_{1} s_{j-1}+$ "

Page 356, line 7: "every linear map" should be "every invertible linear map" 
Page 361, second display of Example 13: Replace with the following:

$$
x^{i} y^{j}= \begin{cases}x^{2 m} y^{2 l}=\left(x^{2}\right)^{m}\left(y^{2}\right)^{l} & \text { if } i, j \text { are even } \\ x^{2 m+1} y^{2 l+1}=\left(x^{2}\right)^{m}\left(y^{2}\right)^{l} x y & \text { if } i, j \text { are odd }\end{cases}
$$

Page 362, Exercise 6: " $k[x, y, z]]^{G}$ " should be " $\mathbb{R}[x, y, z]^{G}$ " in part (d) and again in part (e)

Page 362, part (a) of Exercise 7: " $k[x, y, z]^{G}$ " should be " $\mathbb{R}[x, y, z]^{G}$ "

Page 363, second line of the first display: The third factor of $g$ should be " $(x-y+z) "$

Page 363, part (b) of Exercise 7: " $k[x, y, z]^{G}$ " should be " $\mathbb{R}[x, y, z]^{G}$ " twice one line below the display, once two lines below the display, and once three lines below the display

Page 364, part (b) of Exercise 14: "Use the method of Exercise 13" should be "Use the method of Exercise 12"

Page 367, line -1: At the end of the display, " $\sum_{|\beta|=|G|} R_{G}\left(x^{\beta}\right) u^{\beta}$ " should be

“ $\sum_{|\beta|=|G|} b_{\beta} R_{G}\left(x^{\beta}\right) u^{\beta}$ "

Page 372, part (b) of Exercise 8: "use Exercise 6 and §2” should be "use Exercise 6 and Example 13 of $\S 2$ "

Page 382, part (c) of Exercise 2: "Use Exercise 13" should be "Use Exercise 16"

Page 382, line 2 of Exercise 9: " $b=A \cdot \mathbf{a}$ " should be " $\mathbf{b}=A \cdot \mathbf{a}$ "

Page 383, line 2 of Exercise 12: " $G \cdot \mathbf{b} \cup G \cdot \mathbf{a}-\{\mathbf{a}\}$ ” should be ' $(G \cdot \mathbf{b} \cup G \cdot \mathbf{a}) \backslash\{\mathbf{a}\}$ "

Page 383, line 2 of Exercise 16: "as in Definition 1 of" should be "as in Definition 2 of"

\section{Chapter 8}

Page 394, Exercise 7: "the map (2)" should be "the map (1)"

Page 399 , line -4 : " $\psi$ " should be " $\phi$ " in two places

Page 399 , line $-2:$ “(1: $\left.a_{1}: \cdots: a_{n}\right)$ ” should be “ $\phi\left(a_{1}, \ldots, a_{n}\right)=\left(1: a_{1}: \cdots\right.$ : $\left.a_{n}\right)$ "

Page 404, line 5: " $1 \leq i_{1}<$ " should be " $0 \leq i_{1}<$ "

Page 404, line 2 of Exercise 9: " $f_{i} \in k\left[x_{0}, \ldots, x_{n}\right]$ " should be " $f_{j} \in k\left[x_{0}, \ldots, x_{n}\right]$ "

Page 410, line -1 : " $k\left[x_{1}, \ldots, x_{n}\right]$ " should be " $k\left[x_{0}, \ldots, x_{n}\right]$ " 
Page 414, part (b) of Exercise 13: " $V \backslash V \cap \mathbf{V}(g)$ " should be " $V \backslash(V \cap \mathbf{V}(g))$ "

Page 416, line 2 of Lemma 5: "LM ${ }_{>h}\left(f^{h}\right)$ " should be " $\mathrm{LM}_{>_{h}}\left(f^{h}\right)$ "

Page 416, line -4: "LM ${ }_{>h}\left(f^{h}\right)$ " should be "LM $>_{h}\left(f^{h}\right)$ "

Page 417, equation (2): "LM ${ }_{>h}\left(f^{h}\right)$ " should be "LM $>_{h}\left(f^{h}\right)$ "

Page 417, two lines below equation (2): " $\mathrm{LM}_{>h}\left(g_{i}^{h}\right)$ " should be " $\mathrm{LM}_{>_{h}}\left(g_{i}^{h}\right)$ "

Page 419, second sentence of the proof of Theorem 8: The sentence should be "Applying the proof of part (i) of Proposition 7 with $I$ in place of $\mathbf{I}_{a}(W)$ shows that $Z$ is a projective variety containing $W$."

Page 425, first line following fourth display: "trivial solutions $(0: 0 ; y)$ " should be "trivial solutions $(0,0, y)$ "

Page 429, line -1: "This proves $f \in I^{(0)} \cap \cdots \cap I^{(n)}$ " should be "This proves $f \in I_{n}^{(0)} \cap \cdots \cap I_{n}^{(n)}$,

Page 430, line 17: "Now suppose $f \in I^{(i)}$ " should be "Now suppose $f \in I_{n}^{(i)}$ ",

Page 430, line 20: " $f \in I^{(0)} \cap \cdots \cap I^{(n)}$ " should be " $f \in I_{n}^{(0)} \cap \cdots \cap I_{n}^{(n)}$ "

Page 431, line 2 of the proof of Proposition 8: "Then the proof of Proposition 7" should be "Then Proposition 7"

Page 432, line -1: "point in $\mathbb{P}^{n}$ " should be "point in $\mathbb{P}^{m}$ "

Page 433, line -12: "all have weight $d$ " should be "are all weighted homogeneous of weight $d$ "

Page 443, line below display (9): " $\sigma$ suppose that" should be "suppose that"

Page 445, first display: “ $\left(\begin{array}{llll}a_{0} & a_{1} & a_{2} & a_{3} \\ b_{0} & b_{1} & b_{1} & b_{3}\end{array}\right)$ ” should be “ $\left(\begin{array}{llll}a_{0} & a_{1} & a_{2} & a_{3} \\ b_{0} & b_{1} & b_{2} & b_{3}\end{array}\right)$ "

Page 446, line -3 : " $w_{i j}=\lambda w_{i j}^{\prime}$ " should be " $w_{i j}^{\prime}=\lambda w_{i j}$ "

Page 447, line 5: "through two points" should be "through two distinct points"

Page 447, line 10: "are nonzero, and, hence, determine a line $L$ " should be "are nonzero and distinct, and, hence, determine a unique line $L$ "

Page 448, part (a) of Exercise 5: " $\sum_{i, j=0}^{n} a_{i j} x_{i} x_{i}$ " should be " $\sum_{i, j=0}^{n} a_{i j} x_{i} x_{j}$ "

Page 448, line 1 of Exercise 9: "be nonzero" should be "be nonzero with $Q=\left(a_{i j}\right)$ symmetric"

Page 449, line 3 of Exercise 10: "set of all lines" should be "union of all projective lines"

Page 449, part (a) of Exercise 13: At the end of line 2, add "The image of $F$ is called a projective line in $\mathbb{P}^{n}$."

Page 450, line 5: " $V \subseteq \mathbb{P}^{4}$ " should be " $V \subseteq \mathbb{P}^{9 "}$ 
Page 453, line -6: "nonzero" should be "nonconstant"

Page 456, line 7: " $f=b_{0} z^{m}+\cdots "$ should be " $f=a_{0} z^{m}+\cdots "$

Page 456, line 8: " $b_{0} \in \mathbb{C} \backslash\{0\}$ " should be " $a_{0} \in \mathbb{C} \backslash\{0\}$ "

Page 464, part (a) of Exercise 5: "nonzero polynomial" should be "nonconstant polynomial"

\section{Chapter 9}

Page 472, line 4 of part (b) of Exercise 4: Add "(This is a challenging exercise.)"

Page 481, lines -7 and -6 : "It is easy to generalize this argument and show" should be "By using the discussion following Lemma 4, one can show"

Page 496, Exercise 7: "a $H F_{I}(s)={ }^{a} H P_{I}(s) "$ should be " ${ }^{a} H F_{R / I}(s)={ }^{a} H P_{R / I}(s) "$

Page 496, part (c) of Exercise 10: "with Theorem 15 of Chapter 4, §3" should be "with Proposition 1 of $\S 1$ "

Page 497, part (c) of Exercise 13: "Lemma 5 of $§ 2$ " should be "Lemma 4 of $§ 2$ "

Page 506, part (a) of Exercise 14: "part (a) of the proposition" should be "part (i) of the proposition"

Page 506, part (b) of Exercise 14: "part (b) of the proposition" should be "part (ii) of the proposition"

Page 514, part (a) of Exercise 10: "If $f_{1}, \ldots, f_{s} \in k\left[x_{1}, \ldots, x_{n}\right]$ " should be "If $f, f_{1}, \ldots, f_{s} \in k\left[x_{1}, \ldots, x_{n}\right] "$

Page 535, line -1 : " $k \geq N$ " should be " $i \geq N$ "

Page 536, line 5: " $W \subset$ " should be " $W \subseteq$ "

Page 536, line 1 of Exercise 13: " $W \subset$ " should be " $W \subseteq$ "

Page 537, line 4 of part (d) of Exercise 14: "a curve $L \subseteq \Gamma$ " should be "a curve $\widetilde{L} \subseteq \Gamma^{\prime \prime}$

Page 537, hint to part (b) of Exercise 15: " $\mathrm{BL}_{0} V$ " should be " $\mathrm{Bl}_{0} V$ "

Page 537, part (b) of Exercise 16: " $g(q, t q)=0 "$ should be " $g(t q, q)=0$ "

Page 537, part (c) of Exercise 16: " $g(q, t q)=0 "$ should be " $g(t q, q)=0 "$

\section{Chapter 10}

Page 549, part (a) of Exercise 7: "matrix $M_{3}$ in (3)" should be "matrix $M_{3}$ in (5)"

Page 549, line 2 of part (e) of Exercise 7: The line should be " $x>y>z$ and explain its relation to the matrix $N_{2}$ in Example 8." 
Page 559, line 21: " $H F_{S /\langle\operatorname{LT}(G)\rangle}\left(m^{\prime}\right)<H F_{S / I}\left(m^{\prime}\right)$ " should be " $H F_{S /\langle\operatorname{LT}(G)\rangle}\left(m^{\prime}\right)>$ $H F_{S / I}\left(m^{\prime}\right) "$

Page 564, line 3: “ $-v^{2}+\xi^{2}-\zeta^{2}$ " should be " $v^{2}-\xi^{2}+\zeta^{2}$ "

Page 564, line 10: " $-u \eta^{2}+v \zeta^{2}$ " should be " $u \eta^{2}-v \zeta^{2}$ "

Page 564, line 12: " $-u \xi^{2}+u \zeta^{2}+v \eta^{2}$ " should be " $u \xi^{2}-u \zeta^{2}-v \eta^{2}$ "

Page 564, fourth display: " $-\xi^{2} \zeta^{2}+\eta^{4}+\zeta^{4}$ ” should be " $\xi^{2} \zeta^{2}-\eta^{4}-\zeta^{4}$ ”

Page 564, sixth display: " $-x z+y^{2}+z^{2}$ " should be " $x z-y^{2}-z^{2}$ "

Page 570, first display: On the left, “ $\frac{\operatorname{lcm}\left(\operatorname{LM}\left(f_{i}\right), \operatorname{LM}\left(f_{j}\right)\right.}{\operatorname{LT}\left(f_{j}\right)}$ ” should be “ $\frac{\operatorname{lcm}\left(\operatorname{LM}\left(f_{i}\right), \operatorname{LM}\left(f_{j}\right)\right)}{\operatorname{LT}\left(f_{i}\right)}$ " (two errors)

Page 578, line 3 of first display: "xy" should be " $-x y$ "

Page 578 , line -1 : in two places, " $(x y$ " should be " $(-x y$ "

Page 579, line before last display: " $-x y+y^{2}$ " should be " $x y+y^{2}$ "

Page 582, line 2 of Example 6: " $f_{2}=x y^{2}-x y$ " should be " $f_{2}=x y^{2}+x y$ "

Page 582, first display of Example 6: " $-x\left(x y^{2}-x y\right)$ " should be " $-x\left(x y^{2}+x y\right)$ "

Page 582, line after first display of Example 6: "equal to $\operatorname{LT}\left(f_{1}\right)$ " should be "equal to $-\mathrm{LT}\left(f_{1}\right)$ "

Page 582, third display: " $\mathfrak{i}(\mathbf{g})=x \mathbf{e}_{2}$ " should be "is $(\mathbf{g})=-x \mathbf{e}_{2}$ "

Page 585, line above the second display: " $y \mathbf{e}_{1}-x \mathbf{e}_{1}$ " should be " $y \mathbf{e}_{1}-x \mathbf{e}_{2}$ "

Page 585, line below the second display: " $\mathfrak{s}(\mathbf{h})=x^{2} \mathbf{e}_{2}$ divides $\mathfrak{s}(\mathbf{k})$ " should be “s(k) $=x^{2} \mathbf{e}_{2}$ divides $\mathfrak{s}(\mathbf{h}) "$

Page 585, line below the second display: "a a" should be "a"

Page 586, first line of pseudocode: " $f_{i} \in R$ " should be " $f_{i} \in R, f_{i}$ monic"

Page 590, part (c) of Exercise 1: On the first line, "order on $S$ " should be "order on $R$ "

Page 590, part (c) of Exercise 1: On the second line, "> should be " $>$ POT"

Page 590, part (b) of Exercise 2: Replace with "If we allow $\mathfrak{s}$-reductions and reduction by the syzygy $\mathbf{h}=\left(-f_{2}, f_{1}\right)$, then show that we can reduce $\mathbf{g}$ to $(0,0)$."

Page 590, part (c) of Exercise 2: Replace with "Use Propositions 12 and 14 to explain why the computations in parts (a) and (b) are unnecessary."

Page 591, line 10 of the pseudocode in Exercise 5: " $<$ " should be " $<$ POT"

Page 597, line -10 "Cramer's Rule" should be "Cramer's rule" 